\title{
Contraction of Stress Fibers Extracted from Smooth Muscle Cells: Effects of Varying Ionic Strength*
}

\author{
Shinji DEGUCHI**, Tsubasa S. MATSUI***, Daiki KOMATSU** \\ and Masaaki SATO**,**** \\ ** Department of Bioengineering and Robotics, Tohoku University \\ 6-6-01 Aramaki-Aoba, Sendai 980-8579, Japan \\ E-mail: deguchi@bml.mech.tohoku.ac.jp \\ *** Department of Biomolecular Sciences, Tohoku University \\ ${ }^{* * * *}$ Department of Biomedical Engineering, Tohoku University
}

\begin{abstract}
Actin stress fibers (SFs) play a key role in regulation of cell adhesion, but the biochemical and biophysical properties intrinsic to SFs remain unclear. Here we extracted SFs from rat embryonic smooth muscle cells by deroofing, and evaluated the effects of varying ionic strength and temperature on their intactness. Wash buffers with ionic strength ranging from 90 to $490 \mathrm{mM}$ were prepared, and the extracted SFs were incubated in a buffer with a particular ionic strength for $10 \mathrm{~min}$ or $24 \mathrm{~h}$. Light and electron microscopy revealed that the extracted SFs comprised tightly packed straight bundles at low ionic strengths that became looser and exhibited a ragged pattern at high ionic strengths. The expression of $\alpha$-actinin associated with the extracted SFs decreased with the increase in ionic strength. Unexpectedly, non-muscle myosin II and smooth muscle myosin in the extracted SFs displayed comparable expression levels over the different ionic strengths. ATP-induced contractility was better preserved at low ionic strengths, including the physiological ionic strength of $170 \mathrm{mM}$. The rate of ATP-induced enzymatic activity increased with increase in temperature, but the difference was not statistically significant. These results demonstrate that low ionic strength produces extracted SFs that are more intact with regard to structure and function.
\end{abstract}

Key words: Stress Fibers, Smooth Muscle Cells, Ionic Strength, Contractility

\section{Introduction}

Stress fibers (SFs) play a key role in regulation of cell adhesion ${ }^{(1,2)}$ and tensional homeostasis ${ }^{(2,3)}$. Myosin II (MII), the main constituent of SFs, is considered to be essential to their function. Characterization of the biochemical and biophysical properties of SFs is important with a focus on the involvement of MII activity.

Samples obtained by homogenization of cells into fragments may obscure properties specific to SFs. To overcome this problem, Katoh et al. ${ }^{(5)}$ developed a method for extracting SFs from cultured cells. In this study, ATP-induced contraction of extracted SFs, driven by the actin-MII interaction, was demonstrated. Extracted SFs have been used by our group to characterize their tensile properties ${ }^{(6-8)}$. Phosphate buffer solutions are generally used in the extraction procedure to wash the samples. The ionic strength of the buffers used is typically within the physiological range (i.e., $\sim 150 \mathrm{mM}$ ). Ionic strength, which is a function of the concentration of all ions present, generally affects protein 
function and stability; however, its effect on extracted SFs has not been investigated in previous studies.

In our earlier studies, we modified Katoh's method and prepared alternative wash buffers using imidazole ${ }^{(9-11)}$. Imidazole is a cationic buffer used within the physiological intracellular $\mathrm{pH}$ range. The aim of the present study was to investigate the effect of an imidazole-based buffer when the ionic strength was changed from 90 to $490 \mathrm{mM}$. Since enzymatic activities such as the ATP-consuming contractile process are generally dependent on temperature, the rate of ATP-induced contraction was measured at different temperatures. Our results demonstrated that the use of buffers with low ionic strengths produces SFs that are more intact with regard to structure and function.

\section{Materials and Methods}

\subsection{Cells and solutions}

A7r5 rat embryonic aortic smooth muscle cell lines (ATCC, CRL-1444) were cultured in DMEM (Invitrogen) supplemented with 10\% FBS (JRH Biosciences) and 1\% penicillin-streptomycin (Invitrogen) in a $5 \% \mathrm{CO}_{2}$ incubator at $37^{\circ} \mathrm{C}$. TEA solution used for extraction of SFs contained $2.5 \mathrm{mM}$ triethanolamine (Wako), $1 \mathrm{mM} \mathrm{DTT,} 1 \mu \mathrm{g} / \mathrm{ml}$ leupeptin, and $1 \mu \mathrm{g} / \mathrm{ml}$ pepstatin (Peptide Institute). Wash buffers containing $20 \mathrm{mM}$ imidazole (MP Biomedicals), 2 mM EGTA (Dojindo), and $2.2 \mathrm{mM} \mathrm{MgCl} 2$ (pMg 2.7) were adjusted to an ionic strength of $90,100,123,170,250,330,410$, or $490 \mathrm{mM}$ at pH 7.2 by the addition of $\mathrm{KCl}$ and $\mathrm{HCl}$. The activation buffer containing $20 \mathrm{mM}$ imidazole, $2 \mathrm{mM}$ EGTA, $2.0 \mathrm{mM} \mathrm{MgCl}$ (pMg 2.7), $1 \mathrm{mM} \mathrm{MgATP} \mathrm{(Wako),} \mathrm{and} 2.0 \mathrm{mM} \mathrm{CaCl}_{2}$ (pCa 4.5) was adjusted to an ionic strength of $94 \mathrm{mM}$ at $\mathrm{pH} 7.1$ by the addition of $\mathrm{KCl}$ and $\mathrm{HCl}$. These different ionic strengths cannot be accurately achieved by simply adding water to the solutions. The buffers were prepared considering all the metal ions and ligands present and their equilibrium constants described in Fabiato and Fabiato ${ }^{(12)}$.

\subsection{Extraction of SFs and light microscopy}

Ventral SFs were extracted from A7r5 cells based on a previously described method ${ }^{(9)}$. In brief, cells were washed twice with cold PBS and deroofed by repeated exposure to ice-cold TEA solution. The extracted SFs were incubated in wash buffer with an ionic strength of $90,100,123,170,250,330,410$, or $490 \mathrm{mM}$ at $4^{\circ} \mathrm{C}$ for $24 \mathrm{~h}$. F-actin in the extracted SFs was stained with $3 \mathrm{nM}$ Alexa 488-phalloidin for $1 \mathrm{~min}$ and observed under a microscope (IX-71; Olympus) equipped with a camera (ORCA-R2; Hamamatsu Photonics).

\subsection{Electron microscopy}

For negative staining electron microscopy (EM), the extracted SFs were incubated in wash buffer with an ionic strength of 90,170 , or $490 \mathrm{mM}$ at $4{ }^{\circ} \mathrm{C}$ for $24 \mathrm{~h}$. SFs were then scraped off the dish using a rubber scraper, put on an EM grid with a Formvar support film, stained with $4 \%$ uranyl acetate solution, quickly dried, and observed under an electron microscope (H-7600; Hitachi) at an accelerating voltage of $100 \mathrm{kV}$.

\subsection{SDS-PAGE and immunoblotting}

The extracted SFs were incubated in wash buffer with an ionic strength of 90, 123, 170, $250,330,410$, or $490 \mathrm{mM}$ at $4^{\circ} \mathrm{C}$ for $24 \mathrm{~h}$. For SDS-PAGE, the extracted SFs and intact cells were separately solubilized in SDS sample buffer, boiled for $5 \mathrm{~min}$, and fractionated on $10 \%$ polyacrylamide gels. Equal amounts of total protein $(5 \mu \mathrm{g})$ were loaded in each lane. Gels were stained with Coomassie Brilliant Blue G-250 (CBB; Bio-Rad Laboratories). Samples were transferred onto polyvinylidene difluoride membranes (Millipore) for immunoblotting. Membranes were incubated with an antibody against non-muscle MIIA 
(NMIIA; 1:1000, Covance), non-muscle MIIB (NMIIB; 1:1000, Abcam), smooth muscle myosin (SMM; 1:1000, Abcam), $\alpha$-actinin (monoclonal, 1:1000, Abcam and polyclonal, 1:200, Santa Cruz Biotechnology), fascin (1:200, Santa Cruz Biotechnology), $\alpha$-smooth muscle actin ( $\alpha$-SMA; 1:1000, Abcam), $\beta$-actin (1:5000, Applied Biological Materials), and myosin light chain (MLC; 1:200, Santa Cruz Biotechnology). The primary antibodies were detected using HRP-conjugated goat anti-mouse and goat anti-rabbit secondary antibodies (1:10000, Bio-Rad Laboratories) and enhanced chemiluminescence (Immobilon Western, Millipore).

\subsection{Contractility assay}

Cells were cultured on 18-mm square cover glasses placed in a plastic dish. The SFs were extracted essentially according to the abovementioned method but under 3 different sets of conditions shown in Table 1. Experiments A and B were designed to investigate how buffer treatment time may affect contractility. Experiment $\mathrm{C}$ was designed to investigate the effect of varying temperature. In Experiment A, the extracted SFs were treated with wash buffer having an ionic strength of $90,123,170,250,330,410$, or $490 \mathrm{mM}$ for $24 \mathrm{~h}$; in Experiment B, SFs were treated with buffer having an ionic strength of 90, 123, 170, 250, 330, 410, or $490 \mathrm{mM}$ for $10 \mathrm{~min}$; and in Experiment C, SFs were briefly washed twice with buffer having an ionic strength of $100 \mathrm{mM}$. A perfusion chamber with a channel was made from the cover glass supporting the extracted SFs ${ }^{(9)}$. The chamber was mounted on the microscope stage to capture time-lapse images and was perfused with activation solution from one side of the chamber via capillary action. SFs were reactivated with activation solutions containing MgATP at the following concentrations and temperatures: $2 \mathrm{mM}$ at room temperature $\left(25^{\circ} \mathrm{C}\right)$ for Experiment $\mathrm{A}, 1 \mathrm{mM}$ at $25^{\circ} \mathrm{C}$ for Experiment $\mathrm{B}$, and $1 \mathrm{mM}$ at either 4,25 , or $37^{\circ} \mathrm{C}$ for Experiment C. After reactivation with $\mathrm{MgATP}$ for either $3 \mathrm{~min}$ (Experiment A) or $5 \mathrm{~min}$ (Experiment B), F-actin was stained with $3 \mathrm{nM}$ Alexa 488 conjugated-phalloidin in PBS for $1 \mathrm{~min}$. Data were quantified using ImageJ (NIH).

Table 1 Experimental conditions of contractility assay.

\begin{tabular}{lllll}
\hline Experiment & Ionic strength & $\begin{array}{l}\text { Treatment } \\
\text { time }\end{array}$ & Temperature & {$[\mathrm{MgATP}]$} \\
\hline Experiment A & $90,123,170,250,330,410$, or $490 \mathrm{mM}$ & $24 \mathrm{~h}$ & $25^{\circ} \mathrm{C}$ & $2 \mathrm{mM}$ \\
Experiment B & $90,123,170,250,330,410$, or $490 \mathrm{mM}$ & $10 \mathrm{~min}$ & $25^{\circ} \mathrm{C}$ & $1 \mathrm{mM}$ \\
Experiment C & $100 \mathrm{mM}$ & 2 washes & 4,25, or $37^{\circ} \mathrm{C}$ & $1 \mathrm{mM}$ \\
\hline
\end{tabular}

\section{Results}

\subsection{Effect of varying ionic strength on SF morphology}

The extracted SFs were observed using phase-contrast microscopy (PM), fluorescence microscopy (FM), and EM (Fig. 1). PM revealed extracted SFs with a sharp and straight morphology at an ionic strength of $90 \mathrm{mM}$. Furthermore, SFs with this morphology were found only at ionic strengths below $250 \mathrm{mM}$, and this morphology was absent at high ionic strengths. The SF outlines became less defined as the ionic strength was increased, and at more than $410 \mathrm{mM}$ they were barely observed. Particularly straight actin filaments labeled with fluorescent phalloidin were observed by FM at an ionic strength of $90 \mathrm{mM}$. As the ionic strength increased, the fluorescent actin bundles appeared thicker in width. The loosely packed structure of the actin bundle was particularly prominent at an ionic strength of $490 \mathrm{mM}$. EM showed that at an ionic strength of $90 \mathrm{mM}$, isolated SFs displayed a tightly packed structure with several filamentous constituents bundled together. As the ionic strength increased, the bundles became looser with larger distances between the 
components. At the highest ionic strength examined $(490 \mathrm{mM})$, the continuous arrangement of straight filamentous constituents was no longer found, but rather, loosely packed, punctuate patterns were observed along the length of the isolated SFs. We quantified the bundle thickness of the isolated SFs from two representative EM images and found that the average thickness was $428 \mathrm{~nm}$ (for $90 \mathrm{mM}), 1029 \mathrm{~nm}(170 \mathrm{mM})$, and $2003 \mathrm{~nm}(490 \mathrm{mM})$ (Table 2).
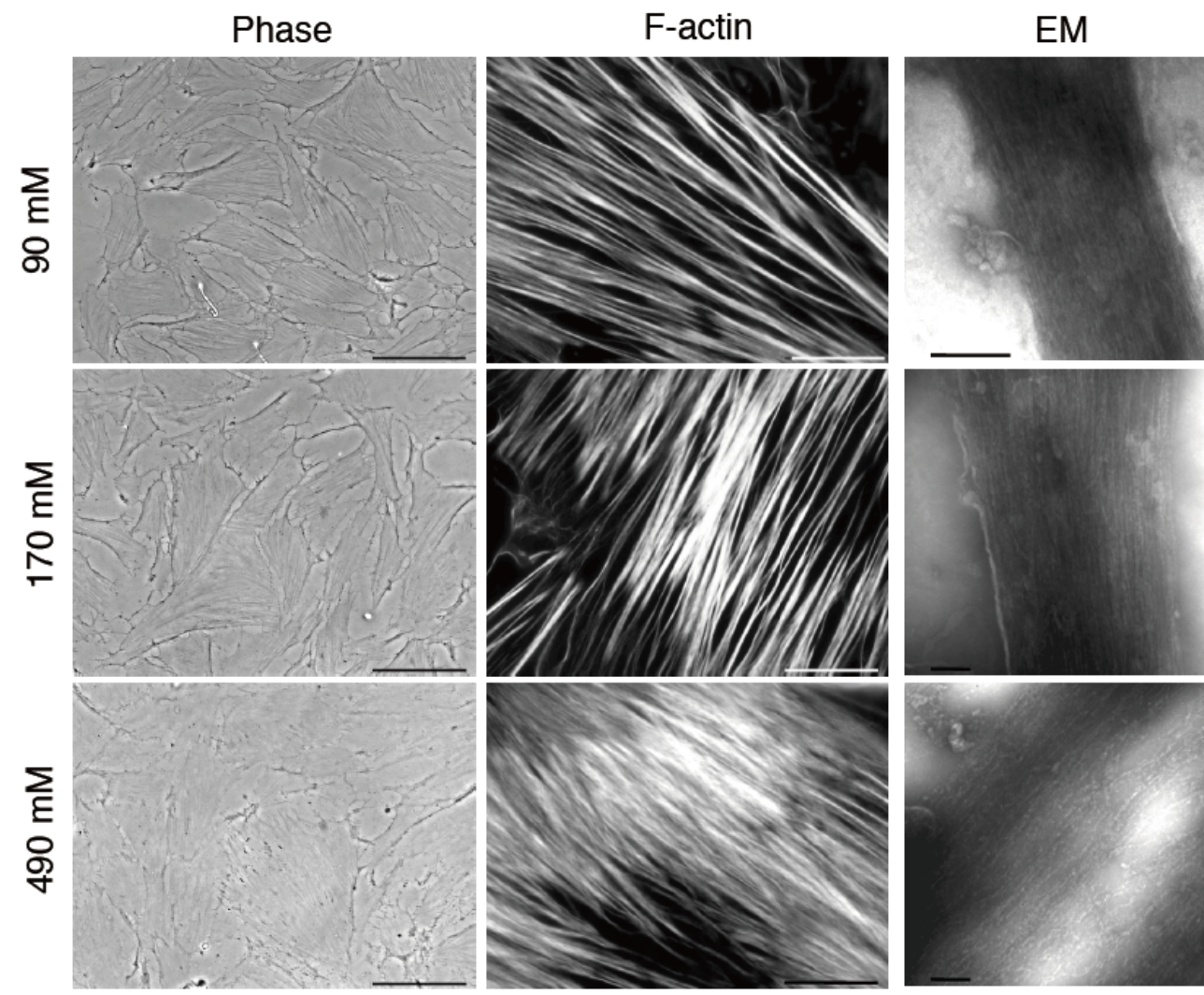

Fig. 1 The effects of varying the ionic strength of buffers on extracted SFs. PM (Phase), multiple cells. FM (F-actin), single cells. EM, single SFs. Scale bars: Phase, $50 \mu \mathrm{m}$; F-actin, $20 \mu \mathrm{m}$; EM, $200 \mathrm{~nm}$.

Table 2 Bundle thickness of individual SFs.

\begin{tabular}{cc}
\hline Ionic strength $(\mathrm{mM})$ & Thickness $(\mathrm{nm})$ \\
\hline 90 & $428 \pm 48$ \\
170 & $1029 \pm 111$ \\
490 & $2003 \pm 272$ \\
\hline
\end{tabular}

Means \pm s.d. $(n=8)$.

\subsection{Effect of varying ionic strength on SF composition}

SDS-PAGE was performed to examine the effects of varying ionic strength on the composition of extracted SFs (Fig. 2). In comparison to whole cell lysates, the extracted SFs contained a darker band around $42 \mathrm{kDa}$, which corresponds to actin. Because samples containing equal amounts of total protein were loaded in each lane, the relative amounts of actin were higher in SFs than in whole cells. A distinct decrease in band intensity was observed at approximately $75 \mathrm{kDa}$ in CBB-stained gels as the ionic strength increased from 90 to $490 \mathrm{mM}$. A total of 8 proteins (NMIIA, NMIIB, SMM, $\alpha$-actinin, fascin, $\alpha$-SMA, 
A

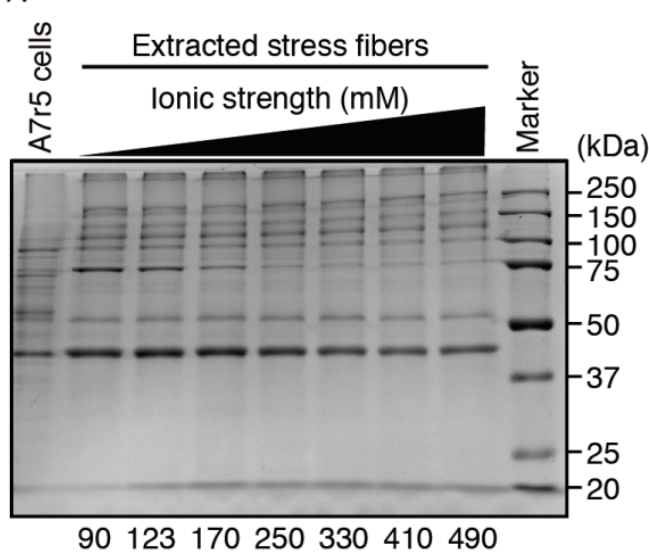

B

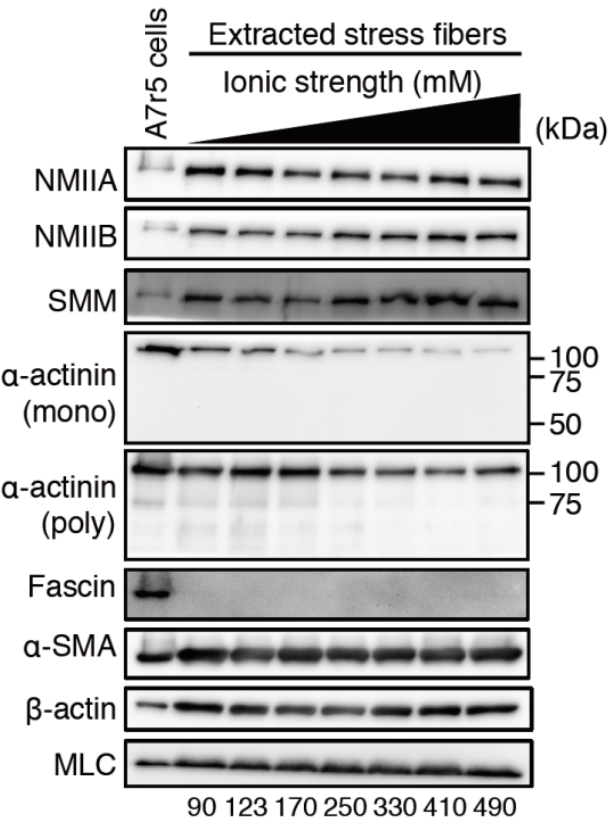

Fig. 2 Comparison of protein concentrations. (A) CBB-stained SDS-PAGE and (B) western blot analyses of whole cell lysates and extracted SFs at ionic strengths ranging from 90 to $490 \mathrm{mM}$. The gel and blots are representative of 2 experiments except for $\alpha$-actinin which was obtained from a single experiment for each antibody. Note that the different blots do not indicate the absolute amount of proteins present in SFs.

$\beta$-actin, and MLC) were identified by immunoblotting. Fascin, a globular F-actin cross-linking protein that is essential for filopodia formation, was only present in whole cell lysates, suggesting that the material we extracted from cells was indeed SFs and not actin bundle filopodia. The expression levels of all proteins, except $\alpha$-actinin, were comparable regardless of the ionic strength. Two different antibodies (monoclonal and polyclonal) were used to detect $\alpha$-actinin, and similar decreases with an increase in ionic strength were detected.

\subsection{Effect of varying ionic strength on SF contractility}

Two separate sets of experiments were performed to induce actin-MII cross-bridge cycling. In Experiment A, $2 \mathrm{mM} \mathrm{MgATP}$ was added to the extracted SFs initially treated with wash buffer of a particular ionic strength for $24 \mathrm{~h}$. In Experiment B, $1 \mathrm{mM} \mathrm{MgATP}$ was added to SFs initially treated with wash buffer of a particular ionic strength for $10 \mathrm{~min}$. Three minutes after the addition of $2 \mathrm{mM} \mathrm{MgATP}$ in Experiment A or $5 \mathrm{~min}$ after the addition of $1 \mathrm{mM} \mathrm{MgATP}$ in Experiment B, PBS containing fluorescent phalloidin was perfused. Thus, ATP-induced shortening was subdued since ATP was removed by PBS perfusions. In Experiment A, majority of the extracted SFs shortened at ionic strengths less than $170 \mathrm{mM}$. It was difficult to observe SFs at high ionic strengths, since there were only a few distinct filamentous structures visible in PM (Fig. 3). At high ionic strengths, only peripheral regions of the cells, which were deroofed in order to expose the SFs to the solutions, shrank in response to ATP. FM confirmed that all SFs shortened and collapsed into a tight mass at ionic strengths below $123 \mathrm{mM}$. Meanwhile, SFs presented as rough, ragged actin structures with almost no shortening at ionic strengths above $410 \mathrm{mM}$. The responses of SFs obtained in Experiment A can be categorized into the following groups: one in which all SFs shortened in response to ATP at low ionic strengths and the other in which some ragged actin structures, which are insensitive to ATP, remained, but 


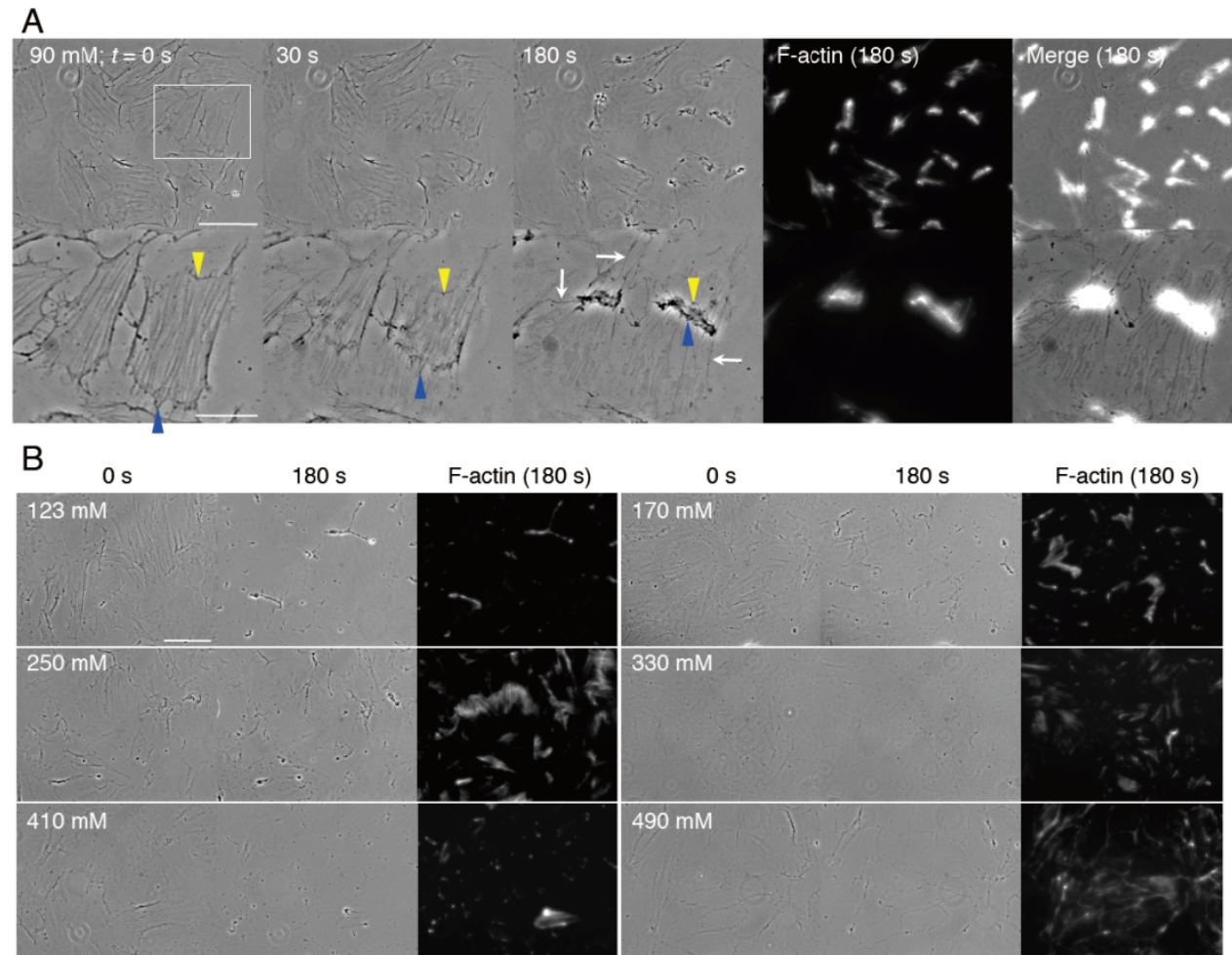

Fig. 3 Active contraction of extracted SFs initially treated with buffers of different ionic strengths for $24 \mathrm{~h}$. (A) Shortening of SFs initially treated with buffer at ionic strength of 90 $\mathrm{mM}$ on the addition of $2 \mathrm{mM} \mathrm{MgATP}$ (at $t=0 \mathrm{~s}$ ). The lower panels show magnified sequential images of a boxed area in the upper panel. Arrowheads indicate the translocation of points on SFs over time to show shortening. Note that SFs shortened to finally collapse into an F-actin-associated mass as visualized by FM. Other filamentous structures (such as those shown by arrows) were left on the substrate after SF shortening. They were not associated with phalloidin-detecting F-actin, and thus were probably the extracellular matrix. (B) Shortening of SFs initially treated with buffers of different ionic strengths. Note that the SFs almost completely shortened at an ionic strength of $123 \mathrm{mM}$ or less, while many non-contractile F-actin structures remained even after $180 \mathrm{~s}$ at high ionic strengths. Scale bars: (A) $50 \mu \mathrm{m}$; magnified images in (A) $20 \mu \mathrm{m}$; (B) $50 \mu \mathrm{m}$.

unidentified peripheral regions of the cell shrank at high ionic strengths. In Experiment B, we quantified the proportion of cells that shortened to completely collapse into a mass within the 5-min interaction with ATP. At an ionic strength of 170, 250, or $330 \mathrm{mM}$, all SFs shrank in response to ATP. However, at an ionic strength of $90,123,410$, or $490 \mathrm{mM}$ only some cells (on average $60 \%-80 \%$ of the total) exhibited a contractile response (Fig. 4 ). SFs in the non-shortened cells initially treated at an ionic strength of 90 or $123 \mathrm{mM}$ displayed a straight morphology. Meanwhile, SFs in the non-shortened cells initially treated at an ionic strength of 410 or $490 \mathrm{mM}$ displayed ragged actin structures with few fibers showing a straight morphology. The responses of SFs obtained in Experiment B, can be categorized into the following 3 groups (Fig. 4B): (I) group in which some straight SFs remain without shortening even in the presence of ATP; (II) group in which all SFs shorten in the presence of ATP; and (III) group in which some ragged actin structures, which are insensitive to ATP, remain.

\subsection{Effect of varying temperature on the shortening rate of SFs}

In Experiment $\mathrm{C}$, the effects of varying temperature on cell contractility was investigated using SFs extracted at an ionic strength of $100 \mathrm{mM}$ which lies within region I of Fig. 4B. Many SFs began to shorten on the addition of $1 \mathrm{mM} \mathrm{MgATP}$ at $t=0$ (Fig. 5). The shortening rate normalized to the initial length of SFs gradually decreased over time 
A

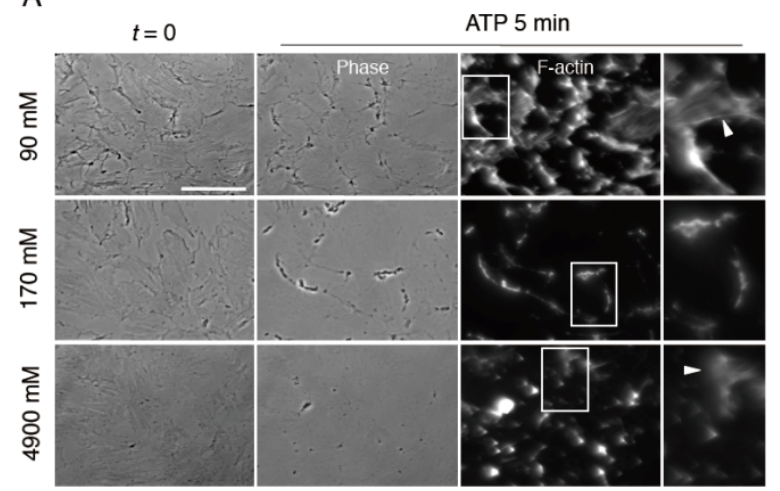

B

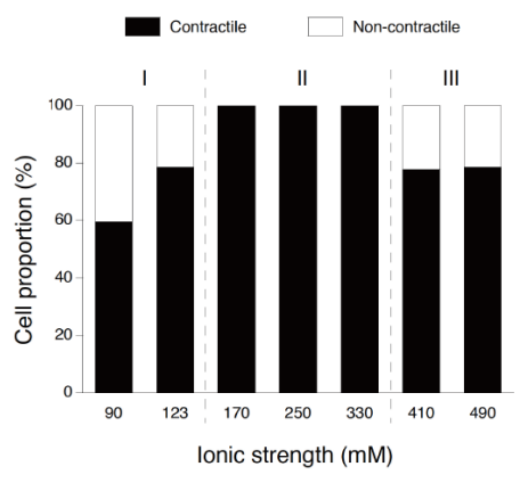

Fig. 4 Active contraction of extracted SFs initially treated with buffers of different ionic strengths for $10 \mathrm{~min}$. (A) Shortening of SFs (initially treated with buffer at ionic strength of 90,170 , or $490 \mathrm{mM}$ ) after the addition of $1 \mathrm{mM} \mathrm{MgATP}$ at $t=0 \mathrm{~s}$. The panels on the right represent a magnified view of the boxed areas. Note that SFs completely shortened at an ionic strength of $170 \mathrm{mM}$, while some SFs (such as those shown by arrow heads) did not shorten and remained unchanged at other ionic strengths. (B) The histogram shows the proportion of contractile and non-contractile cells. The responses of SFs can thus be categorized into 3 groups, denoted as regions I, II, and III.
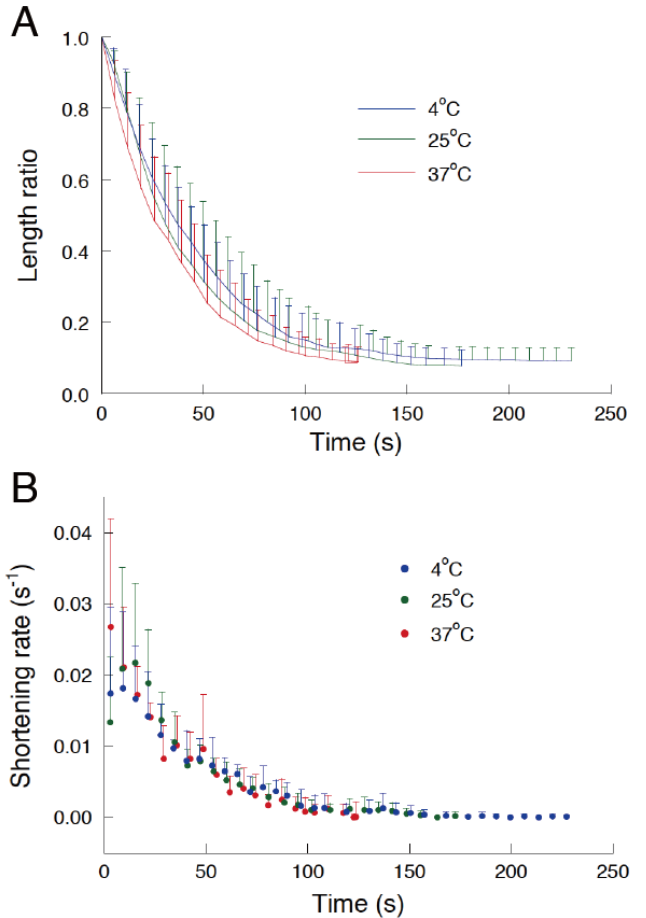

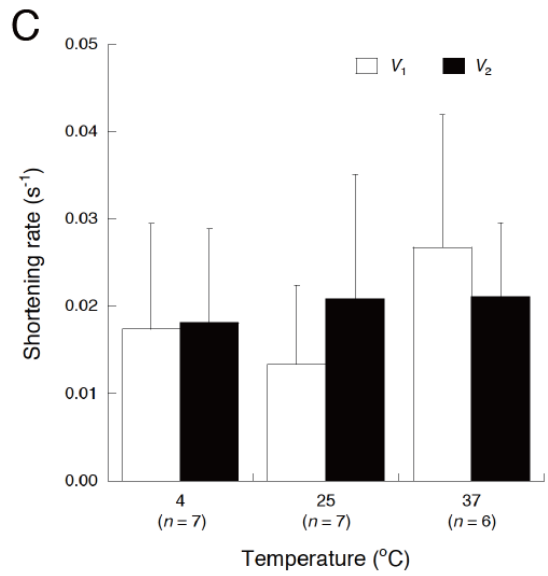

Fig. 5 The effects of temperature on SF contractility. (A) Time-course change in the length of SFs normalized to their initial length. Data are means \pm s.d. ( $n=6-7$ experiments). (B) Time-course change in the shortening rate. (C) Shortening rates measured at $t=0-5 \mathrm{~s}\left(V_{1}\right)$ and 5-10 s $\left(V_{2}\right)$. Data are means + s.d.

(Fig. 5A and 5B), and was therefore quantified over 2 different time intervals. $V_{1}$ and $V_{2}$ were calculated from the rate for $t=0-5 \mathrm{~s}$ and 5-10 s, respectively (Fig. 5C). The average value of the shortening rates tended to increase with an increase in temperature for both $V_{1}$ and $V_{2}$. The differences were analyzed using the Student's $t$-test, but no significant differences were observed between the shortening rates at different temperatures for both $V_{1}$ and $V_{2}$. 


\section{Discussion}

Experiments for SFs in intact cells ${ }^{(2,4,13)}$ are often only suggestive rather than demonstrative of the molecular mechanisms underlying their specific contributions. The inherent mechanisms at the molecular level may be studied with SFs extracted from live cells. A method for mass isolation of functional SFs from cells was introduced by Katoh et al. ${ }^{(5)}$, in which the cell membranes and cytoplasmic constituents were removed by procedures similar to those used in the present study. The absence of the surrounding cytoplasmic structures permits controlled solutions direct access to the extracted SFs that remain on the substrate. However, these in vitro studies may overlook some of the regulatory mechanisms present in intact cells, which could be lost or altered in the extracted systems. Thus, we investigated how structure, molecular composition, and contractility of SFs could differ during the extraction procedure when the ionic strength of the wash buffer and temperature were altered. The imidazole-based buffer we used was a modification of that described by Fabiato and Fabiato ${ }^{(12)}$, taking advantage of accumulated techniques developed for skinned muscle cells or fibers that were first introduced by Natori ${ }^{(14)}$.

Our results suggest that the extracted SFs may be more intact with regard to structure (Fig. 1), composition (Fig. 2), and contractility (Fig. 3) at low ionic strengths. The three experiments (i.e., morphological observation, composition analysis, and contractility measurement) employed the SFs treated with buffer at a particular ionic strength for $24 \mathrm{~h}$. A long exposure time was selected in order to achieve the desired effect. Meanwhile, we examined the contractility of SFs treated with buffer at a particular ionic strength for $10 \mathrm{~min}$ (Fig. 4). The selection of this short exposure time (in Experiment B) was to evaluate the effects of ionic strength within a practical treatment time, i.e., when extracted from cells, SFs are usually exposed to buffers within a few minutes ${ }^{(9)}$. In Experiment B, the extracted SFs exhibited the most extensive shortening at an intermediate ionic strength corresponding to region II (Fig. 4). We speculate that the protein structure of the adhesion-matrix complex and contractility may remain intact in region I. This is consistent with the fact that MII is known to form an anti-parallel folded dimer, suitable for contractile activity, at low or physiological ionic strengths ${ }^{(15,16)}$. It is possible that the SFs were not able to shorten in length because the intact adhesions continued to function as anchors even after extraction. Because the extracted SFs maintained a straight morphology in region I as seen in live cells, they may have been undergoing an isometric contraction (with an unchanged macroscopic length of SFs) in the presence of ATP. At intermediate ionic strengths, all cells shortened to collapse into a mass. This suggests that the adhesion-matrix complex and/or matrix itself may have denatured even during the short treatment period of $10 \mathrm{~min}$, and it was subsequently unable to function as an anchor to prevent shortening.

In contrast, all SFs shortened even at low ionic strengths when treated for $24 \mathrm{~h}$ in Experiment A (Fig. 3). This result suggests that the adhesion-matrix complex and/or matrix itself may have denatured even at the low stabilizing ionic strength when treated for a long time. Fibronectin, a principal constituent of the matrix, has been reported to undergo an ionic strength-dependent change in conformation between a compact and an extended form ${ }^{(17)}$. In addition, disulfide bonds in matrix proteins could be reduced in the extracted state due to the presence of oxygen-removal reagents in the solutions. Thus, further study is required for exclusion of the possible involvement of the matrix and cell adhesions. Development of a novel system for physically isolating functional SFs from the substrate is underway in our group to definitively characterize the contractile properties intrinsic to SFs.

The extracted SFs were packed more loosely at high ionic strengths (Fig. 1). We originally speculated that denaturation of MII may be responsible for this loose packing because of a partial loss of SF contractility (Figs. 3 and 4). However, there was no significant change in the expression of MII (NM2A, NM2B, and SMM) and MLC (Fig. 2). Instead we found that $\alpha$-actinin associated with the extracted SFs decreased in expression 
with an increase in ionic strength. Incubation with the anti- $\alpha$-actinin polyclonal antibody revealed multiple bands including one at approximately $75 \mathrm{kDa}$ in addition to the known band at $105 \mathrm{kDa}$ (Fig. 2). Each band became less intense as the ionic strength increased. The affinity between non-muscle $\alpha$-actinin and actin is known to be significantly reduced in the presence of $\mathrm{Ca}^{2+(18)}$. However, since we removed $\mathrm{Ca}^{2+}$ from wash buffers with EGTA, its involvement in $\alpha$-actinin dissociation is unlikely. Regardless of the mechanism of $\alpha$-actinin dissociation, sarcomere structures might disintegrate at high ionic strengths, resulting in the loss of contractility and the appearance of ragged rather than straight patterns.

One critical point of regulation for contraction and maintenance of SFs is MLC phosphorylation. MLC phosphorylation is required for MII bipolar filament assembly and ATP-induced actin-MII cross-bridge cycling ${ }^{(1)}$. Katoh et al. ${ }^{(5)}$ reported that no MLC phosphorylation was initially detected in SFs isolated from human foreskin fibroblasts. They observed a gradual increase in MLC phosphorylation after the addition of exogenous ATP to the isolated SFs with recombinant Rho-kinase and/or MLCK that phosphorylate MLC. The use of PBS might alter protein activities such as MLC phosphorylation. In contrast, we previously detected mono- and diphosphorylated MLC in the SFs extracted from A7r5 cells using an imidazole-based buffer with an ionic strength of $170 \mathrm{mM}^{(9,10)}$. We did not detect significant monophosphorylation of MLC in the present study at the physiological ionic strength (figure not shown). In the present study, the extracted SFs were washed with buffer for $24 \mathrm{~h}$, whereas in the previous studies they were washed for a short period of less than $1 \mathrm{~min}$. The long wash duration employed in the present study thus somehow decreased phosphorylation.

MgATP $(1 \mathrm{mM})$ was added to the SFs extracted at an ionic strength of $100 \mathrm{mM}$ at different temperatures to evaluate their contractility (Fig. 5). The resulting shortening could be hampered by the adhesion-matrix anchor (Fig. 4). Such physical restrictions may prevent or at least slow the initial shortening. We then focused on cells that were isolated from their surrounding neighbors, and thus, were physically independent of the effect of cell-cell interaction. The shortening rate was quantified at the following time intervals: $V_{1}$ being the initial rate measured for $t=0-5 \mathrm{~s}$ and $V_{2}$ for $5-10 \mathrm{~s}$. Since $V_{1}$ could be more susceptible to retardation due to local physical restrictions, $V_{2}$ might be regarded as being more independent of such surrounding restrictions and could thus be a more accurate measure of contractility. $V_{2}$ increased on an average with an increase in temperature, but the difference was not statistically significant (Fig. 5). Although a temperature-dependent difference exists for the ATPase rate of the MII enzyme itself ${ }^{(19)}$, we did not detect a significant difference at the level of SFs. Thus, while the increase in ionic strength contributed to a positive entropy-driven change, the change in temperature may not have provided a significant enthalpy change in the structure of the extracted SFs.

Peripheral cell regions shrank in response to ATP in both Experiments A and B at all ionic strengths, while SFs shortened only at low ionic strengths (Figs. 3 and 4). The peripheral regions with a relatively rough morphology are distinct from SFs having a clear filamentous appearance. Although we did not identify the peripheral regions, we speculate that because they were labeled with phalloidin, they were the actin network-lined cell membranes left after the extraction procedure. MII and its contractile activity were probably present in the membranes, causing shrinkage of the rough structures on ATP addition (Figs. 3 and 4). The shrinkage of the actin-MII network in the membranes may not require $\alpha$-actinin. On the other hand, $\alpha$-actinin decreased in expression in the extracted SFs at high ionic strengths (Fig. 2), and they exhibited no contractility even with MII activity. Thus, the presence of $\alpha$-actinin or its involvement in maintaining sarcomere integrity is implicated in the shortening of SFs. 


\section{Conclusions}

We investigated the effects of varying the ionic strength of buffers and temperature on SFs extracted from cells. Light microscopy and EM revealed that SFs possessed more straight, packed bundles at low ionic strengths that lost contractility completely when treated with high ionic strength buffers for a long period. We did not detect a significant difference in contractility with a change in temperature at low ionic strengths. These results suggest that the extracted SFs are more intact with regard to structure and function at low ionic strengths, but this conclusion may only apply at the macroscopic level. The present study thus provides practical methodological information that could be useful for investigators interested in performing studies on extracted systems.

\section{Acknowledgments}

The authors thank Hitomi Onodera for assistance with the experiments and Mitsuji Kaji for technical support in EM. This work was supported in part by JSPS KAKENHI Grants (Nos. 21680039 and 22650098 to S.D., 23-7552 to T.S.M., and 20001007 to M.S.) and JST Special Coordination Funds for Promoting Science and Technology (to S.D.).

\section{References}

(1) Chrzanowska-Wodnicka, M. and Burridge, K., Rho-stimulated contractility drives the formation of stress fibers and focal adhesions, Journal of Cell Biology, Vol.133, No.6 (1996), pp.1403-1415.

(2) Deguchi, S., Matsui, T.S., and Iio, K., The position and size of individual focal adhesions are determined by intracellular stress-dependent positive regulation, Cytoskeleton, Vol.68, No.11 (2011), pp.639-651.

(3) Deguchi, S. and Sato, M., Biomechanical properties of actin stress fibers of non-motile cells, Biorheology, Vol.46, No.2 (2009), pp.93-105.

(4) Kaunas, R. and Deguchi, S., Multiple roles for myosin II in tensional homeostasis under mechanical unloading, Cellular Molecular Bioengineering, Vol.4, No.2 (2011), pp.182-191.

(5) Katoh, K., Kano, Y., Masuda, M., Onishi, H., and Fujiwara, K., Isolation and contraction of the stress fiber, Molecular Biology of the Cell, Vol.9, No.7 (1998), pp.1919-1938.

(6) Deguchi, S., Ohashi, T., and Sato, M., Intracellular stress transmission through actin stress fiber network in adherent vascular cells, Molecular \& Cellular Biomechanics Vol.2, No.4 (2005), pp.205-216.

(7) Deguchi, S., Ohashi, T., and Sato, M., Tensile properties of single stress fibers isolated from cultured vascular smooth muscle cells, Journal of Biomechanics, Vol.39, No.14 (2006), pp.2603-2610.

(8) Matsui, T.S., Deguchi, S., Sakamoto, N., Ohashi, T., and Sato, M., A versatile micro-mechanical tester for actin stress fibers isolated from cells, Biorheology, Vol.46, No.2 (2009), pp.401-415.

(9) Matsui, T.S., Ito, K., Kaunas, R., Sato, M., and Deguchi, S., Actin stress fibers are at a tipping point between conventional shortening and rapid disassembly at physiological levels of MgATP, Biochemical and Biophysical Research Communications, Vol.395, No.3 (2010), pp.301-306.

(10) Matsui, T.S., Kaunas, R., Kanzaki, M., Sato, M., and Deguchi, S., Non-muscle myosin II induces disassembly of actin stress fibres independently of myosin light chain dephosphorylation, Interface Focus, Vol.1, No.5 (2011), pp.754-766.

(11) Deguchi, S., Matsui, T.S., and Sato, M., Simultaneous contraction and buckling of stress fibers in individual cells, Journal of Cellular Biochemistry, Vol.113, No.3 (2012), 
pp.824-832.

(12) Fabiato, A. and Fabiato, F. Calculator programs for computing the composition of the solutions containing multiple metals and ligands used for experiments in skinned muscle cells, Journal de Physiologie, Vol.75, No.5 (1979), pp.463-505.

(13) Nagayama, K. and Matsumoto, T., Estimation of single stress fiber stiffness in cultured aortic smooth muscle cells under relaxed and contracted states: Its relation to dynamic rearrangement of stress fibers, Journal of Biomechanics, Vol.43, No.8 (2010), pp.1443-1449.

(14) Natori, R., The property and contraction process of isolated myofibrils, Jikeikai Medical Journal, Vol.1, No.1 (1954), pp.119-126.

(15) Scholey, J.M., Taylor, K.A., and Kendrick-Jones, J., Regulation of non-muscle myosin assembly by calmodulin-dependent light chain kinase, Nature, Vol.287, No.5779 (1980), pp.233-235.

(16) Trybus, K.M. and Lowey, S., Conformational states of smooth muscle myosin Effects of light chain phosphorylation and ionic strength, Journal of Biological Chemistry, Vol.259, No.13 (1984), pp.8564-8571.

(17) Erickson, H.P. and Carrell, N.A., Fibronectin in extended and compact conformations. Electron microscopy and sedimentation analysis, Journal of Biological Chemistry, Vol.258, No.23 (1983), pp.14539-14544.

(18) Burridge, K. and Feramisco, J.R. Nonmuscle $\alpha$-actinins are calcium-sensitive actin-binding proteins, Nature, Vol.294, No.5841 (1981), pp.565-567.

(19) Levy, H.M., Sharon, N., Ryan, E.M., and Koshland, D.E. Jr., Effect of temperature on the rate of hydrolysis of adenosine triphosphate and inosine triphosphate by myosin with and without modifiers. Evidence for a change in protein conformation. Biochimica et Biophysica Acta, Vol.56 (1962), pp.118-126. 